\title{
Geometric Analogy and Products of Vectors in $n$ Dimensions
}

\author{
Leonardo Simal Moreira \\ UniFOA - Centro Universitário de Volta Redonda, Volta Redonda, Brazil \\ Email: simal.leonardo@terra.com.br, leonardo.moreira@foa.org.br
}

Received November 12, 2012; revised December 21, 2012; accepted December 30, 2012

\begin{abstract}
The cross product in Euclidean space $I R^{3}$ is an operation in which two vectors are associated to generate a third vector, also in space $I R^{3}$. This product can be studied rewriting its basic equations in a matrix structure, more specifically in terms of determinants. Such a structure allows extending, for analogy, the ideas of the cross product for a type of the product of vectors in higher dimensions, through the systematic increase of the number of rows and columns in determinants that constitute the equations. So, in a $n$-dimensional space with Euclidean norm, we can associate $n-1$ vectors and to obtain an $n$-th vector, with the same geometric characteristics of the product in three dimensions. This kind of operation is also a geometric interpretation of the product defined by Eckman [1]. The same analogies are also useful in the verification of algebraic properties of such products, based on known properties of determinants.
\end{abstract}

Keywords: Cross Product; Space IR ${ }^{n}$; Determinants; Geometric Analogy; Eckman’s Product

\section{Introduction}

In the Euclidean space $I R^{3}$, the cross product of two vectors $\boldsymbol{u}$ and $\boldsymbol{v}$ is the vector designated by the symbol $[\boldsymbol{u v}]$, and defined for the following conditions [2]:

a) The norm of vector $[\boldsymbol{u v}]$, symbolized for $\mid[\boldsymbol{u v}]$, is given for

$$
|[\mathbf{u} v]|=|\boldsymbol{u}||\boldsymbol{v}| k,
$$

where $k=\operatorname{sen} \alpha$, being $\alpha$ the angle between the vectors $\boldsymbol{u}$ and $\boldsymbol{v}$.

b) The vector $[\boldsymbol{u v}]$ is perpendicular simultaneously to the vectors $\boldsymbol{u}$ and $\boldsymbol{v}$ :

$$
\begin{aligned}
& {[\boldsymbol{u v}] \cdot \boldsymbol{u}=0,} \\
& {[\boldsymbol{u v}] \cdot \boldsymbol{v}=0 .}
\end{aligned}
$$

As a consequence of $b),[\boldsymbol{u v}]$ is the normal vector to the plane defined for the vectors $\boldsymbol{u}$ and $\boldsymbol{v}$ (Figure 1), if these are linearly independent vectors. Considering $[\boldsymbol{u v}]=(p, q, r)$, then $\beta: p x+q y+r z+c=0$, where $c=-p a_{1}-q a_{2}-r a_{3}$, represents the equation of the plane $\beta$ in a Cartesian coordinate system $\left(A\left(a_{1}, a_{2}, a_{3}\right)\right.$ is a point in $I R^{3}$ and $A \in \beta$ ).

If $\boldsymbol{u}$ and $\boldsymbol{v}$ are linearly dependent vectors, then

$$
[\mathbf{u v}]=\mathbf{0} \text {, }
$$

where the symbol $\mathbf{0}$ represents the null vector. c) The vector $[\boldsymbol{u v}]$ is oriented in relation to the vectors $\boldsymbol{u}$ and $\boldsymbol{v}$ just as, in right-handed coordinate system, the $z$-axis it is oriented in relation to the $x$-axis and $y$-axis.

d) The volume $V_{3}$ of parallelepiped defined for the vectors $\boldsymbol{u}, \boldsymbol{v}$ and $[\boldsymbol{u v}]$ is the square of the number |[uv]| (Figure 2):

$$
V_{3}=|[\boldsymbol{u v}]|^{2}
$$

The equalities (2), (3) and (5) are equivalent to those given in a Definition 1 found in [3].

In this paper, it is shown that it is possible, through simple analogies with the case in the space $I R^{3}$, to extend the ideas of the cross product to the space $I R^{4}$, and more generally, to the space $I R^{n}$. The characteristics of the cross product in $I R^{3}$ are maintained in higher dimensions.

\section{Matrix Structure of [uv]}

The initial reasoning for the extension of the ideas of the cross product is the fact that their basic expressions can be represented in the form of determinants.

In an orthogonal coordinate system, representing the vectors $\boldsymbol{u}$ and $\boldsymbol{v}$ in terms of 3-tuples $\boldsymbol{u}=\left(u_{1}, u_{2}, u_{3}\right)$ and $\boldsymbol{v}=\left(v_{1}, v_{2}, v_{3}\right)$, the vector [uv] can be obtained starting from the development of the symbolic determinant 


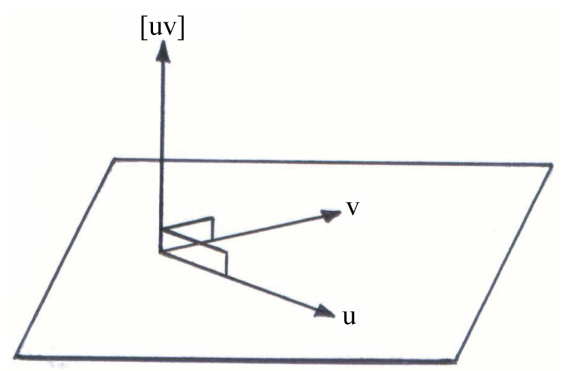

Figure 1. $[u v]$ is the normal vector to the plane defined for the vectors $u$ and $v$.

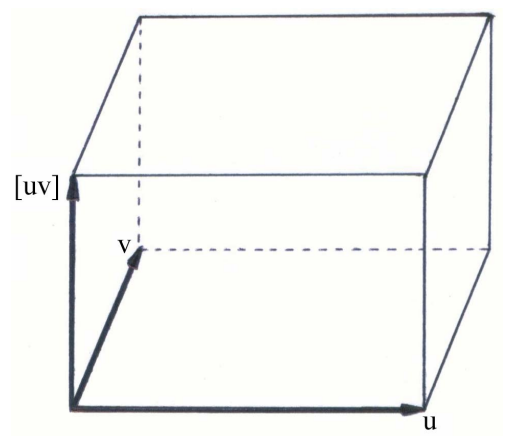

Figure 2. Parallelepiped defined for the vectors $u, v$ and $[u v]$.

$$
[\boldsymbol{u v}]=\left|\begin{array}{ccc}
\hat{e}_{1} & \hat{e}_{2} & \hat{e}_{3} \\
u_{1} & u_{2} & u_{3} \\
v_{1} & v_{2} & v_{3}
\end{array}\right|
$$

where $\hat{e}_{1}=(1,0,0), \hat{e}_{2}=(0,1,0), \hat{e}_{3}=(0,0,1)$ are the vectors of orthonormal basis in $I R^{3}$.

The development of the Equation (6) leads to the vector form:

$$
[\boldsymbol{u v}]=\left|\begin{array}{ll}
u_{2} & u_{3} \\
v_{2} & v_{3}
\end{array}\right| \hat{e}_{1}-\left|\begin{array}{ll}
u_{1} & u_{3} \\
v_{1} & v_{3}
\end{array}\right| \hat{e}_{2}+\left|\begin{array}{cc}
u_{1} & u_{2} \\
v_{1} & v_{2}
\end{array}\right| \hat{e}_{3},
$$

and the norm of vector $[\boldsymbol{u v}]$ is calculated with the definition of Euclidean norm, resulting in

$$
|[\boldsymbol{u v}]|=\left(\left|\begin{array}{ll}
u_{2} & u_{3} \\
v_{2} & v_{3}
\end{array}\right|^{2}+\left|\begin{array}{cc}
u_{1} & u_{3} \\
v_{1} & v_{3}
\end{array}\right|^{2}+\left|\begin{array}{ll}
u_{1} & u_{2} \\
v_{1} & v_{2}
\end{array}\right|^{2}\right)^{\frac{1}{2}},
$$

an equivalent format to

$$
|[\boldsymbol{u v}]|=\left|\begin{array}{cc}
u_{2} & u_{3} \\
v_{2} & v_{3}
\end{array}\right| \quad(-1)\left|\begin{array}{cc}
u_{1} & u_{3} \\
v_{1} & v_{3}
\end{array}\right| \mid \begin{array}{cc}
u_{1} & u_{2} \\
v_{1} & v_{2}
\end{array} \|^{\frac{1}{2}}
$$

In Equation (1),

$$
k=\operatorname{sen} \alpha=\left|\begin{array}{cc}
1 & \cos \alpha \\
\cos \alpha & 1
\end{array}\right|^{\frac{1}{2}},(0 \leq \alpha \leq \pi),
$$

and combining the Equations (9) and (10), we obtain

$$
\begin{aligned}
& \left.\left|\begin{array}{cc}
u_{2} & u_{3} \\
v_{2} & v_{3}
\end{array}\right| \quad(-1)\left|\begin{array}{cc}
u_{1} & u_{3} \\
v_{1} & v_{3}
\end{array}\right|\left|\begin{array}{cc}
u_{1} & u_{2} \\
v_{1} & v_{2}
\end{array}\right|\right|^{\frac{1}{2}} \\
& =|\boldsymbol{u}||\boldsymbol{v}|\left|\begin{array}{cc}
1 & \cos \alpha \\
\cos \alpha & 1
\end{array}\right|^{\frac{1}{2}}
\end{aligned}
$$

Equation (11) will be used as starting point for the analogies developed in the remaining of this work.

\section{Extension of the Cross Product to the Euclidean Space $I R^{4}$}

Consider three vectors in Euclidean space $I R^{4}$, represented in terms of quadruples $\boldsymbol{v}_{1}=\left(v_{11}, v_{12}, v_{13}, v_{14}\right)$, $\boldsymbol{v}_{2}=\left(v_{21}, v_{22}, v_{23}, v_{24}\right)$ and $\boldsymbol{v}_{3}=\left(v_{31}, v_{32}, v_{33}, v_{34}\right)$. Let $\hat{e}_{1}=(1,0,0,0), \hat{e}_{2}=(0,1,0,0), \hat{e}_{3}=(0,0,1,0)$ and $\hat{e}_{4}=(0,0,0,1)$ be the vectors of orthonormal basis in $I R^{4}$.

It is possible to develop an equivalent product to (1), through simple extension of ideas and increase of dimensions. In space $I R^{3}$, two vectors $\boldsymbol{u}$ and $\boldsymbol{v}$ generate a third vector whose norm is proportional to the product of the norms of the generating vectors, being the proportionality constant related to the angle between $\boldsymbol{u}$ and $\boldsymbol{v}$. In space $I R^{4}$, three vectors $\boldsymbol{v}_{1}, \boldsymbol{v}_{2}$ and $\boldsymbol{v}_{3}$ generate a fourth vector whose norm is proportional to the product of the norms of the generating vectors, being the proportionality constant related to the angles between the vectors $\boldsymbol{v}_{1}$ and $\boldsymbol{v}_{2}, \boldsymbol{v}_{1}$ and $\boldsymbol{v}_{3}, \boldsymbol{v}_{2}$ and $\boldsymbol{v}_{3}$.

In symbolic terms, this product of vectors in Euclidean space $I R^{4}$ is obtained from the development of the determinant

$$
\boldsymbol{h}=\left[\boldsymbol{v}_{1} \boldsymbol{v}_{2} \boldsymbol{v}_{3}\right]=\left|\begin{array}{cccc}
\hat{e}_{1} & \hat{e}_{2} & \hat{e}_{3} & \hat{e}_{4} \\
v_{11} & v_{12} & v_{13} & v_{14} \\
v_{21} & v_{22} & v_{23} & v_{24} \\
v_{31} & v_{32} & v_{33} & v_{34}
\end{array}\right|,
$$

so that

$$
|\boldsymbol{h}|=\left|\boldsymbol{v}_{1}\right|\left|\boldsymbol{v}_{2}\right|\left|\boldsymbol{v}_{3}\right| \bar{k}
$$

with

$$
\bar{k}=\left|\begin{array}{ccc}
1 & \cos \alpha_{12} & \cos \alpha_{13} \\
\cos \alpha_{21} & 1 & \cos \alpha_{23} \\
\cos \alpha_{31} & \cos \alpha_{32} & 1
\end{array}\right|^{\frac{1}{2}}
$$


and the conditions $\left(\begin{array}{l}\alpha_{12}+\alpha_{13}+\alpha_{21} \leq 2 \pi, \\ \alpha_{12} \leq \alpha_{13}+\alpha_{21}, \\ \alpha_{13} \leq \alpha_{12}+\alpha_{21}, \\ \alpha_{21} \leq \alpha_{12}+\alpha_{13} .\end{array}\right)$.

The equal sign in the conditions on the angles, given in (14), is justified for the case of coplanar vectors.

In Equation (14), $\cos \alpha_{i j}=\frac{\boldsymbol{v}_{i} \cdot \boldsymbol{v}_{j}}{\left|\boldsymbol{v}_{i}\right|\left|\boldsymbol{v}_{j}\right|}$ represents the angle between two of the generating vectors of $\boldsymbol{h}$, and naturally $\cos \alpha_{i j}=\cos \alpha_{j i}$, so that $\bar{k}^{2}$ is the determinant of a symmetric matrix.

The equivalent in space $I R^{4}$ of Equation (11) is (see the Equation (15) below):

The characteristics of the product $[\boldsymbol{u v}]$ in space $I R^{3}$ are conserved for $\boldsymbol{h}$ in space $I R^{4}$ :

a) The norm of $\boldsymbol{h}$ is proportional to the product $\left|\boldsymbol{v}_{1}\right|\left|\boldsymbol{v}_{2}\right|\left|\boldsymbol{v}_{3}\right|$. It is sufficient to develop the determinants in Equation (15) to verify the identity.

b) The vector $\boldsymbol{h}$ is perpendicular to each one of the vectors $\boldsymbol{v}_{1}, \boldsymbol{v}_{2}$ and $\boldsymbol{v}_{3}$. The term "perpendicular" should be interpreted here as only in the sense that the scalar product $\boldsymbol{h} \cdot \boldsymbol{v}_{i}$ results null.

PROOF: The elements of the 1st row of the determinant that represents the norm of $\boldsymbol{h}$ are the same values as their own cofactors. It is known that the sum of the products of the elements of a row for the cofactors of the elements corresponding of other row (inner product) in a determinant results in zero (Cauchy's Determinant Theorem), that is, $\boldsymbol{h} \cdot \boldsymbol{v}_{1}=\boldsymbol{h} \cdot \boldsymbol{v}_{2}=\boldsymbol{h} \cdot \boldsymbol{v}_{3}=0$.

It is also noted that $\boldsymbol{h}$ is the normal vector to the hyperplane that contains $\boldsymbol{v}_{1}, \boldsymbol{v}_{2}$ and $\boldsymbol{v}_{3}$. Being

$\boldsymbol{h}=h_{1} \hat{e}_{1}+h_{2} \hat{e}_{2}+h_{3} \hat{e}_{3}+h_{4} \hat{e}_{4}$, then

$\mathcal{H}: h_{1} x_{1}+h_{2} x_{2}+h_{3} x_{3}+h_{4} x_{4}+\bar{c}=0$, where

$\bar{c}=-h_{1} a_{1}-h_{2} a_{2}-h_{3} a_{3}-h_{4} a_{4}$, represents the Cartesian equation of hyperplane $\mathcal{H}\left(A\left(a_{1}, a_{2}, a_{3}, a_{4}\right)\right.$ is a point in $I R^{4}$ and $\left.A \in \mathcal{H}\right)$.

c) The vector $\boldsymbol{h}$ is oriented in relation to the vectors $\boldsymbol{v}_{1}, \boldsymbol{v}_{2}$ and $\boldsymbol{v}_{3}$ just as the vector $-\hat{e}_{4}$ in relation to $\hat{e}_{1}$, $\hat{e}_{2}$ and $\hat{e}_{3}$.

d) The content of parallelotope defined for the vectors $\boldsymbol{v}_{1}, \boldsymbol{v}_{2}, \boldsymbol{v}_{3}$ and $\boldsymbol{h}$ is the square of number $|\boldsymbol{h}|$.

PROOF: With effect, the determinant to the left in
Equation (15) represents the number $|\boldsymbol{h}|$. In this way, $|\boldsymbol{h}|^{2}$ is the determinant whose rows are formed by the vectors $\boldsymbol{h}, \boldsymbol{v}_{1}, \boldsymbol{v}_{2}$ and $\boldsymbol{v}_{3}$, representing the content of parallelotope (4-parallelepiped) that has the four vectors as edges linearly independents [4].

\section{Product of $n-1$ Vectors in Euclidean space $I R^{n}$}

Consider $n-1$ vectors in Euclidean space $I R^{n}$, represented in terms of $n$-tuples, such that

$$
\begin{aligned}
& \boldsymbol{v}_{1}=\left(v_{11}, v_{12}, \cdots, v_{1, n}\right), \boldsymbol{v}_{2}=\left(v_{21}, v_{22}, \cdots, v_{2, n}\right) \\
& , \cdots, \boldsymbol{v}_{n-1}=\left(v_{n-1,1}, v_{n-1,2}, \cdots, v_{n-1, n}\right)
\end{aligned}
$$

The product $\boldsymbol{H}=\left[\boldsymbol{v}_{1} \boldsymbol{v}_{2} \cdots \boldsymbol{v}_{n-1}\right]$ in space $I R^{n}$ is a vector perpendicular simultaneously to all the $\boldsymbol{v}_{i}(1 \leq i \leq n-1)$ and whose norm is given by the formula

$$
|\boldsymbol{H}|=\left|\boldsymbol{v}_{1}\right|\left|\boldsymbol{v}_{2}\right| \cdots\left|\boldsymbol{v}_{n-1}\right| K,
$$

with

$$
K=\left|\begin{array}{cccc}
1 & \cos \alpha_{1,2} & \cdots & \cos \alpha_{1, n-1} \\
\cos \alpha_{2,1} & 1 & \cdots & \cos \alpha_{2, n-1} \\
\vdots & \vdots & \ddots & \vdots \\
\cos \alpha_{n-1,1} & \cos \alpha_{n-1,2} & \cdots & 1
\end{array}\right|^{\frac{1}{2}}
$$

It is observed that this form is equivalent to the products of vectors defined by [1], and cited in [5,6], namely (using the same symbols as in [6]), that a cross product satisfies the axioms:

(A1) $\left\langle P\left(a_{1}, \cdots, a_{r}\right), a_{i}\right\rangle=0,(1 \leq i \leq r)$,

(A2) $\left\|P\left(a_{1}, \cdots, a_{r}\right)\right\|^{2}=\operatorname{det}\left(\left\langle a_{i}, a_{j}\right\rangle\right)$,

where $\|a\|^{2}=\langle a, a\rangle$.

These preliminary definitions can be formalized starting from the following proposition.

PROPOSITION: Let $n-1$ vectors be in space $I R^{n}$, with inner product and Euclidean norm. Consider also that the vectors are represented by $n$-tuples such that

$$
\begin{aligned}
& \boldsymbol{v}_{1}=\left(v_{11}, v_{12}, \cdots, v_{1, n}\right), \boldsymbol{v}_{2}=\left(v_{21}, v_{22}, \cdots, v_{2, n}\right) \\
& , \cdots, v_{n-1}=\left(v_{n-1,1}, v_{n-1,2}, \cdots, v_{n-1, n}\right)
\end{aligned}
$$

Being $\cos \alpha_{i j}$ the angle between the $i$-th vector $v_{i}$ and the $j$-th vector $\boldsymbol{v}_{j}$, the following equality is true (see the Equation (18) below):

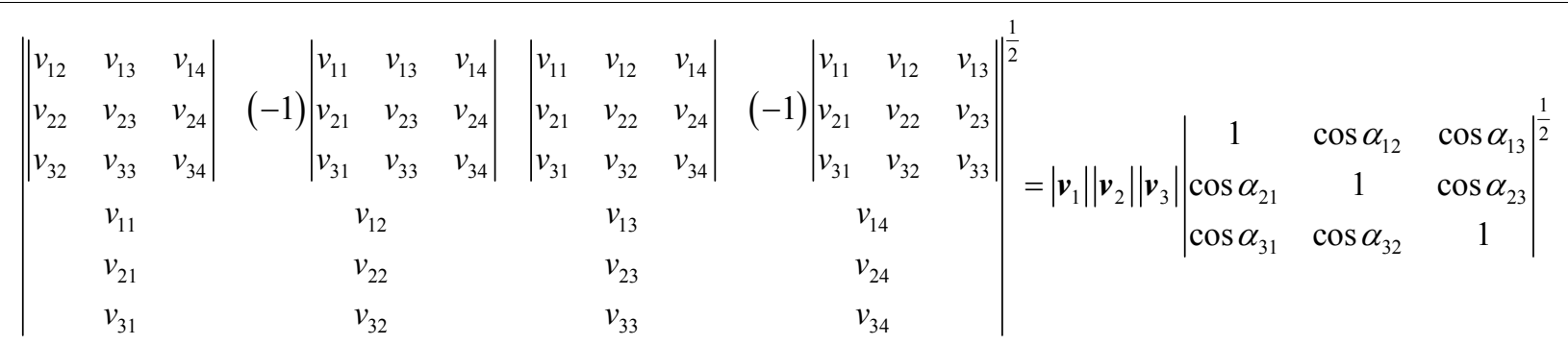




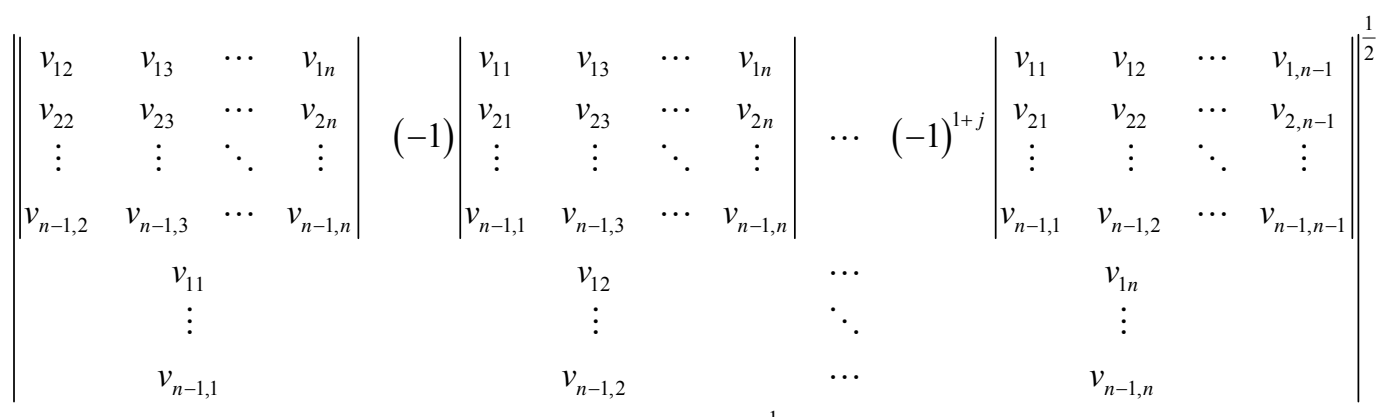

$$
\begin{aligned}
& =\left|\boldsymbol{v}_{1}\right|\left|\boldsymbol{v}_{2}\right| \cdots\left|\boldsymbol{v}_{n-1}\right|\left|\begin{array}{cccc}
1 & \cos \alpha_{12} & \cdots & \cos \alpha_{1, n-1} \\
\cos \alpha_{21} & 1 & \cdots & \cos \alpha_{2, n-1} \\
\vdots & \vdots & \ddots & \vdots \\
\cos \alpha_{n-1,1} & \cos \alpha_{n-1,2} & \cdots & \cos \alpha_{n-1, n-1}
\end{array}\right|^{\frac{1}{2}}
\end{aligned}
$$

PROOF: Consider $n-1$ unit vectors $\boldsymbol{u}_{i}(1 \leq i \leq n-1)$ in space $I R^{n}$, with inner product and Euclidean norm. Consider also that each $\boldsymbol{u}_{i}$ represents the unit vector in the same direction of $\boldsymbol{v}_{i}$ given in the Equation (18), so that

$$
\mathbf{u}_{i}=\frac{\boldsymbol{v}_{i}}{\left|\boldsymbol{v}_{i}\right|} .
$$

If the unit vectors are represented by $n$-tuples such that

$$
\begin{aligned}
& \left|\begin{array}{cccc}
u_{12} & u_{13} & \cdots & u_{1 n} \\
u_{22} & u_{23} & \cdots & u_{2 n} \\
\vdots & \vdots & \ddots & \vdots \\
u_{n-1,2} & u_{n-1,3} & \cdots & u_{n-1, n}
\end{array}\right| \\
& \\
& u_{11}
\end{aligned}
$$

Starting from Equation (20), Equation (18) can be demonstrated. With effect, multiplying both members of (20) for $\left(\left|\boldsymbol{v}_{1}\right|\left|\boldsymbol{v}_{2}\right| \cdots\left|\boldsymbol{v}_{n-1}\right|\right)^{2}$, the determinant to the left will have their rows orderly and appropriately multiplied

$$
\begin{aligned}
& \boldsymbol{u}_{1}=\left(u_{11}, u_{12}, \cdots, u_{1 n}\right), \boldsymbol{u}_{2}=\left(u_{21}, u_{22}, \cdots, u_{2 n}\right) \\
& , \cdots, \boldsymbol{u}_{n-1}=\left(u_{n-1,1}, u_{n-1,2}, \cdots, u_{n-1, n}\right)
\end{aligned}
$$

being $\boldsymbol{u}_{i} \cdot \boldsymbol{u}_{j}$ the inner product between the $i$-th unit vector $\boldsymbol{u}_{i}$ and the $j$-th unit vector $\boldsymbol{u}_{j}$, can be grouped, based on the properties presented in (A2), the components of $\boldsymbol{u}_{i}$ in the following identity, which is true for values of $n \geq 3$ :

by each one of $\left|\boldsymbol{v}_{i}\right|$, and since $\left|\boldsymbol{v}_{\boldsymbol{i}}\right| \boldsymbol{u}_{i}=\boldsymbol{v}_{i}$, is obtained the corresponding determinant of Equation (18).

Representing, for convenience,

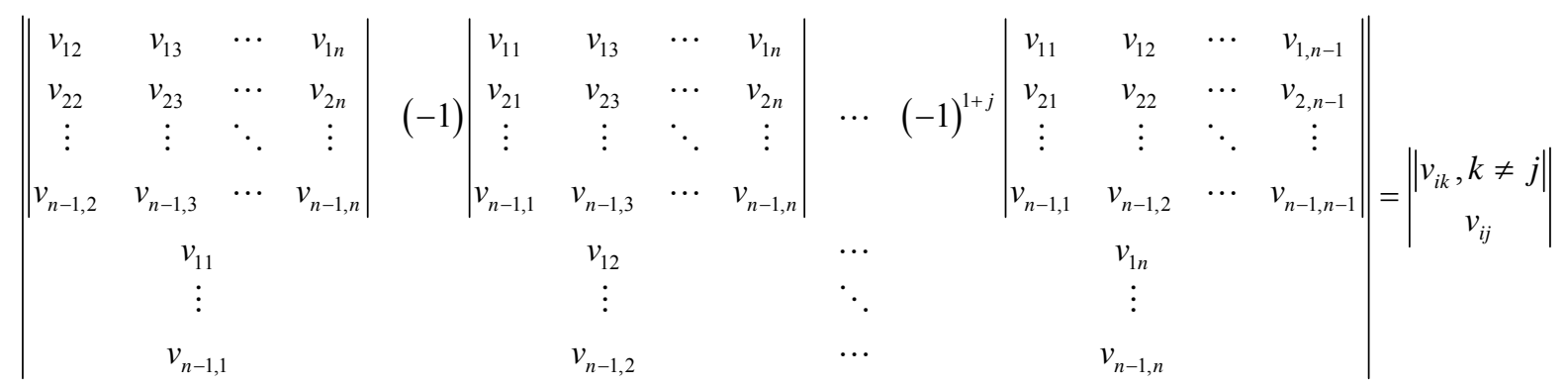

\footnotetext{
$(1 \leq i \leq n-1,1 \leq j \leq n, 1 \leq k \leq n)$
} 
we have that:

$$
\left(\left|\boldsymbol{v}_{1}\right|\left|\boldsymbol{v}_{2}\right| \cdots\left|\boldsymbol{v}_{n-1}\right|\right)^{2}\left|\begin{array}{c}
u_{i k}, k \neq j \\
u_{i j}
\end{array}\right|=\left|\begin{array}{c}
v_{i k}, k \neq j \| \\
v_{i j}
\end{array}\right| .
$$

In relation to the determinant to the right in Equation (20), it is sufficient to observe that $\left|\boldsymbol{u}_{i}\right|=1$, therefore $\cos \alpha_{i j}=\boldsymbol{u}_{i} \cdot \boldsymbol{u}_{j}$, that is:

$$
\begin{aligned}
& \left|\begin{array}{cccc}
1 & \boldsymbol{u}_{1} \cdot \boldsymbol{u}_{2} & \cdots & \boldsymbol{u}_{1} \cdot \boldsymbol{u}_{n-1} \\
\boldsymbol{u}_{2} \cdot \boldsymbol{u}_{1} & 1 & \cdots & \boldsymbol{u}_{2} \cdot \boldsymbol{u}_{n-1} \\
\vdots & \vdots & \ddots & \vdots \\
\boldsymbol{u}_{n-1} \cdot \boldsymbol{u}_{1} & \boldsymbol{u}_{n-1} \cdot \boldsymbol{u}_{2} & \cdots & 1
\end{array}\right| \\
& =\left|\begin{array}{cccc}
1 & \cos \alpha_{12} & \cdots & \cos \alpha_{1, n-1} \\
\cos \alpha_{21} & 1 & \cdots & \cos \alpha_{2, n-1} \\
\vdots & \vdots & \ddots & \vdots \\
\cos \alpha_{n-1,1} & \cos \alpha_{n-1,2} & \cdots & \cos \alpha_{n-1, n-1}
\end{array}\right|
\end{aligned}
$$

With such considerations, it is demonstrated that

$$
\begin{aligned}
& \left|\begin{array}{ccc}
v_{i k}, k \neq j \mid \\
v_{i j}
\end{array}\right|=\left(\left|\boldsymbol{v}_{1}\right|\left|\boldsymbol{v}_{2}\right| \cdots\left|\boldsymbol{v}_{n-1}\right|\right)^{2} \\
& \times\left|\begin{array}{cccc}
1 & \cos \alpha_{12} & \cdots & \cos \alpha_{1, n-1} \\
\cos \alpha_{21} & 1 & \cdots & \cos \alpha_{2, n-1} \\
\vdots & \vdots & \ddots & \vdots \\
\cos \alpha_{n-1,1} & \cos \alpha_{n-1,2} & \cdots & \cos \alpha_{n-1, n-1}
\end{array}\right|,
\end{aligned}
$$

and the square root of Equation (23) shows that Equation (18) is true.

Equation (18) is the equivalent $n$-dimensional of the Equations (11) and (15), validating the extension of cross product. The geometric properties of $\boldsymbol{H}$ are conserved in $n$ dimensions:

a) The norm of $\boldsymbol{H}$ is proportional to the product $\left|\boldsymbol{v}_{1}\right|\left|\boldsymbol{v}_{2}\right| \cdots\left|\boldsymbol{v}_{n-1}\right|$, being the proportionality constant $K$ associated to the angles between the vectors $\boldsymbol{v}_{i}$.

PROOF: The proof consists of the own demonstration of the Equation (18).

b) The vector $\boldsymbol{H}$ is "perpendicular" to each one of the vectors $\boldsymbol{v}_{1}, \boldsymbol{v}_{2}, \cdots, \boldsymbol{v}_{n-1}$.

PROOF: The elements of the 1st row of the determinant that represents the norm of $\boldsymbol{H}$ are the same values as their own cofactors. In agreement with Cauchy's Determinant Theorem, the sum of the products of the elements of a row for the cofactors of the elements corresponding of another row (inner product) in a determinant results in zero, that is, $\boldsymbol{H} \cdot \boldsymbol{v}_{1}=\boldsymbol{H} \cdot \boldsymbol{v}_{2}=\cdots=\boldsymbol{H} \cdot \boldsymbol{v}_{n-1}=0$.

It is also noted that $\boldsymbol{H}$ is the normal vector to the hyperplane that contains $\boldsymbol{v}_{1}, \boldsymbol{v}_{2}, \cdots, \boldsymbol{v}_{n-1}$. Being

$\boldsymbol{H}=H_{1} \hat{e}_{1}+H_{2} \hat{e}_{2}+\cdots+H_{n} \hat{e}_{n}$, then

$\mathcal{H}_{n}: H_{1} x_{1}+H_{2} x_{2}+\cdots+H_{n} x_{n}+C=0$, where

$C=-H_{1} a_{1}-H_{2} a_{2}-\cdots-H_{n} a_{n}$, represents the Cartesian equation of hyperplane $\mathcal{H}_{n}\left(A\left(a_{1}, a_{2}, \cdots, a_{n}\right)\right.$ is a point in $I^{n}$ and $A \in \mathcal{H}^{n}$ ).

c) The vector $\boldsymbol{H}$ is oriented in relation to the vectors $\boldsymbol{v}_{1}, \boldsymbol{v}_{2}, \cdots, \boldsymbol{v}_{n-1}$ just as the vector $(-1)^{n+1} \hat{e}_{n}$ is oriented in relation to $\hat{e}_{1}, \hat{e}_{2}, \cdots, \hat{e}_{n-1}$.

d) The content of parallelotope defined for the vectors $\boldsymbol{v}_{1}, \boldsymbol{v}_{2}, \cdots, \boldsymbol{v}_{n-1}$ and $\boldsymbol{H}$ is the square of number $|\boldsymbol{H}|$.

PROOF: The determinant to the left in Equation (18) represents the number $|\boldsymbol{H}|$. In this way, $|\boldsymbol{H}|^{2}$ is the determinant whose rows are formed by the vectors $\boldsymbol{H}, \boldsymbol{v}_{1}, \boldsymbol{v}_{2}, \cdots, \boldsymbol{v}_{n-1}$, representing the content of parallelotope ( $n$-parallelepiped) that has the $n$ vectors as edges linearly independents [4].

\section{Conclusions}

The possibility to represent the equations of the definition of cross product in the space $I R^{3}$ in terms of determinants allows the extension of the concept of the product of vectors for higher dimensions, systematically increasing rows and columns to the determinants.

Through basic properties of determinants, it is shown that the characteristics of the cross product are conserved in $n$ dimensions, for any value of $n$, since such properties are not modified by the increment or decrease of rows and columns to these determinants.

Other geometric properties can be verified, as the relationship between the cross product and area, because just as the number $|[\boldsymbol{u v}]|$ is related to areas of triangles and parallelograms, the number $|\boldsymbol{H}|$ is related to contents of simplex and parallelotopes, in an equivalent way to Cayley-Menger determinant [7,8].

Although this work has given emphasis to the geometric properties of the product of vectors in the space $I R^{n}$, it indirectly shows that their algebraic properties are also similar to those valid ones in space $I R^{3}$, for instance:

(C1) If $\boldsymbol{w}_{i}$ is any vector in space $I R^{n}$ for $i=1,2, \cdots, n$, then
a) $[w w \cdots w]=0$;
b) $\left[0 w_{1} \cdots w_{n-1}\right]=\mathbf{0}$;
c) $\left[w_{1} w_{2} \cdots \mathbf{0}\right]=\mathbf{0}$;
d) $\left[\boldsymbol{w}_{1} \boldsymbol{w}_{2} \cdots \boldsymbol{w}_{n}\right]=\mathbf{0}$ if any of vectors $\boldsymbol{w}_{i}$ is the null vector.

(C2) The position change among two vectors in the product $\boldsymbol{W}=\left[\boldsymbol{w}_{1} \boldsymbol{w}_{2} \cdots \boldsymbol{w}_{n}\right]$ results in the vector $-\boldsymbol{W}$.

(C3) If $w_{i}$ is any vector in space $I R^{n}$ for $i=1,2, \cdots, n$, and $a \in I R$, then
a) $\left[\left(a w_{1}\right) w_{2} \cdots w_{n}\right]=\left[w_{1}\left(a w_{2} \cdots w_{n}\right)\right]$;
b) $\left[\left(a \boldsymbol{w}_{1}\right) \boldsymbol{w}_{2} \cdots \boldsymbol{w}_{n}\right]=a\left[\boldsymbol{w}_{1} \boldsymbol{w}_{2} \cdots \boldsymbol{w}_{n}\right]$.

These and other algebraic properties, including the distributive property of the product in relation to the sum of 
vectors, are verified easily by the application of the convenient rules on determinants to the matrix structure of product of vectors.

The analogies developed appear still for the possibility of new extensions associated to the concept of products of vectors, such as eventual developments that are related to a type of equivalent $n$-dimensional of the concept of curl, for example.

\section{REFERENCES}

[1] B. Eckmann, "Stetige Lösungen Linearer Gleichungssysteme," Commentarii Mathematici Helvetici, Vol. 15, 1943, pp. 318-339. doi:10.1007/BF02565648

[2] N. Efimov, "Elementos de Geometria Analítica," Cultura Brasileira, São Paulo, 1972.

[3] A. Elduque, "Vector Cross Products," Talk Presented at the Seminario Rubio de Francia of the Universidad de
Zaragoza on April 12004.

[4] S. Lipschutz and M. Lipson, “Álgebra Linear,” Bookman, Porto Alegre, 2008.

[5] R. Brown and A. Gray, "Vector Cross Products," Commentarii Mathematici Helvetici, Vol. 42, 1967, pp. 222236. doi:10.1007/BF02564418

[6] A. Gray, "Vector Cross Products on Manifolds," University of Maryland, College Park, 1968.

[7] P. Gritzmann and V. Klee, "On the Complexity of Some Basic Problems in Computational Convexity II. Volume and Mixed Volumes," In: T. Bisztriczky, P. McMuffen, R. Schneider and A. W. Weiss, Eds., Polytopes: Abstract, Convex and Computational, Kluwer, Dordrecht, 1994, p. 29.

[8] D. M. Y. Sommerville, "An Introduction to the Geometry of $n$ Dimensions," Dover, New York, 1958, p. 124. 